\title{
Evolutionary Multi-Objective Optimization: Basic Concepts and Some Applications in Pattern Recognition
}

\author{
Carlos A. Coello Coello \\ CINVESTAV (Evolutionary Computation Group) \\ Departamento de Computación \\ Av. IPN No. 2508, Col. San Pedro Zacatenco \\ México, D.F. 07360, Mexico \\ ccoello@cs.cinvestav.mx
}

\begin{abstract}
This paper provides a brief introduction to the so-called multi-objective evolutionary algorithms, which are bio-inspired metaheuristics designed to deal with problems having two or more (normally conflicting) objectives. First, we provide some basic concepts related to multi-objective optimization and a brief review of approaches available in the specialized literature. Then, we provide a short review of applications of multi-objective evolutionary algorithms in pattern recognition. In the final part of the paper, we provide some possible paths for future research in this area, which are promising, from the author's perspective.
\end{abstract}

\section{Introduction}

In the real world, there are many problems in which it is desirable to optimize two or more objective functions at the same time. These are known as multiobjective optimization problems (MOPs), and their solution involves finding not one, but a set of solutions that represent the best possible trade-offs among the objective functions being optimized. Such trade-offs constitute the so-called Pareto optimal set, and their corresponding objective function values form the so-called Pareto front.

A number of mathematical programming techniques have been developed to solve MOPs [1. However, they have several limitations, from which the most important are that they tend to be very susceptible to the specific features of the MOP being solved (e.g., the shape or continuity of the Pareto front), and that they normally generate a single solution per run. Such limitations have motivated the development of alternative approaches from which metaheuristic 1 have been, with no doubt, the most popular and effective choice available so far (see for example [3]). From the many metaheuristics in current use, Evolutionary

\footnotetext{
${ }^{1}$ A metaheuristic is a high level strategy for exploring search spaces by using different methods [2]. Metaheuristics have both a diversification (i.e., exploration of the search space) and an intensification (i.e., exploitation of the accumulated search experience) procedure.
} 
Algorithms (EAs) are, clearly, the most popular in today's specialized literature. EAs are inspired on the "survival of the fittest" principle from Darwin's evolutionary theory 4, and simulate the evolutionary process in a computer, as a way to solve problems. EAs have become very popular as multi-objective optimizers because of their ease of use (and implementation) and generality (e.g., EAs are less sensitive than mathematical programming techniques to the initial points used for the search and to the specific features of a MOP). EAs have also an additional advantage: since they are population-based techniques, it is possible for them to manage a set of solutions at a time, instead of only one, as normally done by traditional mathematical programming techniques. This allows EAs to generate several elements from the Pareto optimal set in a single run.

The first Multi-Objective Evolutionary Algorithm (MOEA) was proposed in the mid-1980s by David Schaffer [5]. However, it was until the mid-1990s that MOEAs started to attract serious attention from researchers. Nowadays, it is possible to find applications of MOEAs in practically all domains 2

The rest of this paper is organized as follows. In Section 2, we provide some basic multi-objective optimization concepts required to make this paper selfcontained. An introduction to evolutionary algorithms is provided in Section 3 . Section 4 contains a brief description of the main MOEAs in current use. In Section 5, a short review of some applications of MOEAs in three pattern recognition tasks (image segmentation, feature selection and classification) is provided. Section [6] indicates some potential paths for future research in this area. Finally, the main conclusions of this paper are provided in Section 7.

\section{Basic Concepts}

We are interested in solving problems of the typ 3 :

$$
\operatorname{minimize} \boldsymbol{f}(\boldsymbol{x}):=\left[f_{1}(\boldsymbol{x}), f_{2}(\boldsymbol{x}), \ldots, f_{k}(\boldsymbol{x})\right]
$$

subject to:

$$
\begin{gathered}
g_{i}(\boldsymbol{x}) \leq 0 \quad i=1,2, \ldots, m \\
h_{i}(\boldsymbol{x})=0 \quad i=1,2, \ldots, p
\end{gathered}
$$

where $\boldsymbol{x}=\left[x_{1}, x_{2}, \ldots, x_{n}\right]^{T}$ is the vector of decision variables, $f_{i}: \mathbb{R}^{n} \rightarrow \mathbb{R}, i=$ $1, \ldots, k$ are the objective functions and $g_{i}, h_{j}: \mathbb{R}^{n} \rightarrow \mathbb{R}, i=1, \ldots, m, j=1, \ldots, p$ are the constraint functions of the problem.

To describe the concept of optimality in which we are interested, we will introduce next a few definitions.

\footnotetext{
${ }^{2}$ The author maintains the EMOO repository, which currently contains over 5800 bibliographic references related to evolutionary multi-objective optimization. The EMOO repository is located at: http://delta.cs.cinvestav.mx/ ccoello/EMOO/

${ }^{3}$ Without loss of generality, we will assume only minimization problems.
} 
Definition 1. Given two vectors $\boldsymbol{x}, \boldsymbol{y} \in \mathbb{R}^{k}$, we say that $\boldsymbol{x} \leq \boldsymbol{y}$ if $x_{i} \leq y_{i}$ for $i=1, \ldots, k$, and that $\boldsymbol{x}$ dominates $\boldsymbol{y}$ (denoted by $\boldsymbol{x} \prec \boldsymbol{y}$ ) if $\boldsymbol{x} \leq \boldsymbol{y}$ and $\boldsymbol{x} \neq \boldsymbol{y}$.

Definition 2. We say that a vector of decision variables $\boldsymbol{x} \in \mathcal{X} \subset \mathbb{R}^{n}$ is nondominated with respect to $\mathcal{X}$, if there does not exist another $\boldsymbol{x}^{\prime} \in \mathcal{X}$ such that $\boldsymbol{f}\left(\boldsymbol{x}^{\prime}\right) \prec \boldsymbol{f}(\boldsymbol{x})$.

Definition 3. We say that a vector of decision variables $\boldsymbol{x}^{*} \in \mathcal{F} \subset \mathbb{R}^{n}(\mathcal{F}$ is the feasible region) is Pareto-optimal if it is nondominated with respect to $\mathcal{F}$.

Definition 4. The Pareto Optimal Set $\mathcal{P}^{*}$ is defined by:

$$
\mathcal{P}^{*}=\{\boldsymbol{x} \in \mathcal{F} \mid \boldsymbol{x} \text { is Pareto-optimal }\}
$$

Definition 5. The Pareto Front $\mathcal{P F}^{*}$ is defined by:

$$
\mathcal{P} \mathcal{F}^{*}=\left\{\boldsymbol{f}(\boldsymbol{x}) \in \mathbb{R}^{k} \mid \boldsymbol{x} \in \mathcal{P}^{*}\right\}
$$

We thus wish to determine the Pareto optimal set from the set $\mathcal{F}$ of all the decision variable vectors that satisfy (2) and (3). Note however that in practice, not all the Pareto optimal set is normally desirable (e.g., it may not be desirable to have different solutions that map to the same values in objective function space) or achievable.

\section{A Short Introduction to Evolutionary Algorithms}

Although the origins of evolutionary algorithms (EAs) can be traced back to the early 1930s [6], it was until the 1960s that the three main types of EAs were developed: genetic algorithms [7, evolution strategies [8] and evolutionary programming 9].

EAs are very suitable for solving multi-objective optimization problems because they operate on a set of solutions (called population), which allows them to generate several elements of the Pareto optimal set in a single run (contrasting with mathematical programming techniques, which normally generate a single nondominated solution per execution). Additionally, EAs are less susceptible to the discontinuity and the shape of the Pareto front, which is another important advantage over traditional mathematical programming methods 3 .

Multi-objective Evolutionary Algorithms (MOEAs) extend traditional EAs in two main aspects:

- Selection Mechanism: In MOEAs, the aim is to select nondominated solutions and not the solutions with the highest fitness. Additionally, and according to the definition of Pareto optimality, all the nondominated solutions in a population are normally considered as equally good.

- Diversity Maintenance: MOEAs require a mechanism that preserves diversity and avoids convergence to a single solution (this will eventually happen because of stochastic noise, if an EA is run for a sufficiently long time). 
Regarding selection, several approaches have been adopted over the years, going from simple linear aggregating functions [10] and population-based schemes 5] to ranking approaches based on Pareto optimality [1112] and schemes based on performance measures [13].

Diversity has also been a popular research topic, and a wide variety of methods are currently available to maintain diversity in the population of an EA, including fitness sharing and niching [14 15], clustering [16 17], geographically-based schemes [18, and the use of entropy [1920, among others.

A third component of modern MOEAs is elitism, which normally consists of using an external archive (called a "secondary population") that can interact (or not) in different ways with the main (or "primary") population of a MOEA. Although the main goal of this archive is to store the nondominated solutions generated throughout the search, it has also been used to maintain diversity [21. The approximation of the Pareto optimal set produced by a MOEA can be found in the final contents of this external archive and this is normally the result reported as the outcome of a MOEA's execution.

\section{Multi-Objective Evolutionary Algorithms}

Although there is a wide variety of MOEAs available in the specialized literature, only a handful of them are in wide use. The following are, in the view of the author, the most representative MOEAs in current use:

1. Strength Pareto Evolutionary Algorithm (SPEA): It was conceived as an elegant merge of several MOEAs that were developed during the mid1990s [17. Its main features are an external archive (called the external nondominated set), which stores the nondominated solutions generated during the search. The union of both the external nondominated set and the main population participate in the selection process, during which a strength value is computed for each individual. This strength is proportional to the number of solutions that a certain individual dominates. Then, the fitness of each member of the current population is computed according to the strengths of all the external nondominated solutions that dominate it. The external nondominated set can significantly grow in size, consequently reducing the selection pressure, and slowing down the search. Since this is undesirable, a clustering technique is adopted to prune the contents of the external nondominated set so that its size remains bounded within a certain (user-defined) threshold.

2. Pareto Archived Evolution Strategy (PAES): This approach was introduced in 2000 [21] and is probably the most simple MOEA than can be conceived. It consists of a $(1+1)$ evolution strategy (i.e., a single parent that generates a single offspring) in combination with an external archive that stores the nondominated solutions found so far. This archive is used as a reference set against which each mutated individual is being compared. Such an 
(external) archive adopts a procedure that divides objective function space in a recursive manner. Then, each solution is placed in a certain grid location based on the values of its objectives (which are used as its "geographical location"). A map of such a grid is maintained, indicating the number of solutions that reside in each grid location. When a new nondominated solution is ready to be stored in the archive, but there is no room for it (this is because the size of the external archive is bounded), a check is made on the grid location to which the solution would belong. If this grid location is less densely populated than the most densely populated grid location, then a solution (randomly chosen) from the most populated grid location is deleted in order to allow the storage of the new solution. This aims to redistribute solutions, favoring the less densely populated regions of the Pareto front.

3. Strength Pareto Evolutionary Algorithm 2 (SPEA2): This is a revised version of SPEA, which has three main differences with respect to it [22]: (1) it incorporates a fine-grained fitness assignment strategy which takes into account, for each individual, the number of individuals that dominate it and the number of individuals by which it is dominated; (2) it uses a nearest neighbor density estimation technique which guides the search more efficiently, and (3) it has an enhanced archive truncation method that guarantees the preservation of boundary solutions.

4. Nondominated Sorting Genetic Algorithm II (NSGA-II): This approach is a revised version of one of the earliest MOEAs, called the Nondominated Sorting Genetic Algorithm (NSGA), which was originally introduced in the mid 1990s [1]. The NSGA-II adopts a more efficient ranking procedure than its predecessor. Additionally, it estimates the density of solutions surrounding a particular individual in the population by computing the average distance of two points on either side of this point along each of the objectives of the problem. This value is the so-called crowding distance. During selection, the NSGA-II uses a crowded-comparison operator which takes into consideration both the nondomination rank of an individual in the population and its crowding distance (i.e., nondominated solutions are preferred over dominated solutions, but between two solutions with the same nondomination rank, the one that resides in the less crowded region is preferred). The NSGA-II does not use an external archive as most of the modern MOEAs in current use. Instead, the elitist mechanism of the NSGAII consists of combining the best parents with the best offspring obtained (i.e., a $(\mu+\lambda)$-selection). Due to its clever mechanisms, the NSGA-II is much more efficient (computationally speaking) than its predecessor, and its performance is so good that it has become very popular in the last few years, triggering a significant number of applications, and becoming some sort of landmark against which new MOEAs have to be compared in order to merit publication. 


\section{Multiobjective Evolutionary Algorithm Based on Decomposition} (MOEA/D): This approach was introduced in 2007 [23. It decomposes a problem into a number of scalar optimization sub-problems which are simultaneously optimized. When optimizing each subproblem, only information from its neighboring sub-problems is adopted. This allows this approach to be both very efficient and very effective. MOEA/D is a good example of a MOEA that was able to successfully incorporate concepts from mathematical programming (scalarization functions in this case) into a metaheuristic. Although not as popular as the NSGA-II, MOEA/D has attracted a lot of interest over the years, mainly because of its reputation as a hard-to-defeat MOEA.

Many other MOEAs are currently available (see for example [24 25]), but none of them is widely used in the literature. This, however, has not discouraged algorithm developers who are have now focused their efforts on aspects such as computational efficiency [26] and scalability [13].

\section{Some Applications in Pattern Recognition}

Once can find today a wide variety of applications of MOEAs in pattern recognition. For illustrative purposes only, three types of common applications will be briefly described next:

1. Image Segmentation: We call "segmentation" to the clustering of the pixels of an image based on certain criteria. The output of a segmentation process is usually another image with raw pixel data, which constitutes either the boundary of a region or all the points in the region itself. Segmentation is known to be, in general, a very difficult task. Treated as a multi-objective optimization problem, image segmentation can involve a number of objectives [27]. For example, Bhanu and Lee [28] considered five objectives when applying a genetic algorithm with a linear aggregating function to an image segmentation problem: (1) edge-border coincidence, (2) boundary consistency, (3) pixel classification, (4) object overlap, and (5) object contrast. Shirakawa and Nagao 29], however, considered the minimization of only two objectives (they adopted SPEA2 22 in this case): (1) overall deviation between the data items and their corresponding cluster center (minimizing this objective increases the number of clusters) and (2) edge value, which evaluates the overall summed distances on boundaries between the regions. However, regardless of the objectives adopted, a good motivation for using MOEAs in image segmentation is that they allow the generation of several output images, representing the different trade-offs among the objectives. This gives the user more options to choose from, instead of the single image that is traditionally obtained when using single-objective optimization techniques.

2. Feature Selection: Feature selection refers to the extraction of features for differentiating one class of objects from another. The output of this process 
is a vector of values of the measured features. In this case, when feature selection is treated as a multi-objective problem, the two most common objectives are: (1) the minimization of the number of features and (2) the minimization of the error associated with the solution obtained. For example, Hamdani et al. 30] considered as their second objective the classification error of a nearest neighbor (1-NN) classifier 31] in an application in which the NSGA-II [32] was used as their search engine. Similarly, Morita et al. 33. adopted as their second objective a validity index that measures the quality of the clusters formed. In this case, the authors were dealing with a handwritten word recognition task in which the original NSGA [11] was used as their search engine. It is also possible to introduce additional objectives related, for example, to cost 34] or some problem-specific characteristics 35. This illustrates the flexibility that MOEAs provide when applied to pattern recognition tasks.

3. Classification: This is a process in which each input value is placed into a class (from several available) based on the information provided by its descriptors. When treated as a multi-objective problem, classification normally involves objectives such as minimizing the complexity of the classifier (e.g., its number of rules) while maximizing its accuracy (i.e., the error of the classifier). However, other objectives such as the generality of the rules, their understandability or their complexity can also be adopted. For example, Iglesia et al. 36.37] maximized confidence and coverage of the rules in a partial classification problem (the so-called nugget discovery task), in which the NSGA-II 32] was adopted as the search engine. In contrast, Bandyopadhyay et al. 38] considered three objectives: minimize (1) the number of misclassified training points and (2) the number of hyperplanes, and maximize (3) the product classwise correct recognition rates. The search engine adopted in this case, was as an approach introduced by the authors, and called the constrained elitist multiobjective genetic algorithm based classifier (CEMOGA-Classifier). One of the advantages of the use of MOEAs in classification is that they can overcome problems commonly associated to traditional (i.e., single-objective) classifiers, such as overfitting/overlearning and ignoring smaller classes.

\section{Potential Areas for Further Research}

As we have seen, MOEAs have been applied to several problems in pattern recognition. However, there are other possible paths for future research that may be worth exploring. For example:

- Integration: The development of fully automated pattern recognition systems should be a long-term goal related to the use of MOEAs in this area. Such systems should be applicable to different databases with minimum (or no) human intervention. The development of such systems could require the hybridization of MOEAs with other techniques (e.g., fuzzy logic and/or machine learning techniques) as well as the design of new architectures that 
allow an efficient and effective integration of different types of approaches throughout the different stages involved in a pattern recognition process (see for example [39], in which an automatic image pattern recognition system based on genetic programming [40] is proposed for the classification of medical images). However, MOEAs are an excellent choice for this sort of task because of their capability to deal with conflicting objectives.

- Efficiency: MOEAs are certainly powerful optimization tools, but they normally have a relatively high computational cost because of the number of objective function evaluations that they require in order to produce reasonably good results. This is particularly important in tasks such as image segmentation in which each objective function evaluation will be normally costly. In order to deal with this problem, it is possible to adopt approaches such as fitness approximation [41, parallelization [42, or surrogate methods 43. It is worth mentioning, however, that the incorporation of such techniques in pattern recognition tasks, although promising, is still scarce.

- Use of other Metaheuristics: A number of other bio-inspired metaheuristics have become increasingly popular in the last few years 44, and most of them have been applied to pattern recognition tasks, although their use has been fairly limited until now. The following are representative examples of these new metaheuristics:

- Particle Swarm Optimization (PSO): It was proposed in the mid1990s [45] and simulates the movements of a flock of birds which seek food. In PSO, the behavior of each individual (or particle) is affected by either the best local (i.e., within a certain neighborhood) or the best global (i.e., with respect to the entire population or swarm) individual. Although this approach also adopts a population and a fitness measure, unlike EAs, it allows individuals to benefit from their past experiences. PSO has been used in some pattern recognition tasks (see for example 46]), but not much in a multi-objective form.

- Artificial Immune Systems (AIS): From a computational point of view, our immune system can be seen as a highly parallel intelligent system that is able to learn and retrieve previous knowledge (in other words, it has "memory"), while solving highly complex recognition and classification tasks. These interesting features motivated the development of the so-called artificial immune systems in the early 1990s 4748]. This sort of approach has been used in a wide variety of tasks, including classification and pattern recognition in general (see for example [49]). However, as in the previous case, the use of AISs as multi-objective solvers of pattern recognition problems is still rare.

- Ant Colony Optimization (ACO): It is inspired on the behavior shown by colonies of real ants which deposit a chemical substance on the 
ground called pheromone [50]. The pheromone influences the behavior of the ants: they tend to take those paths in which there is a larger amount of pheromone. Therefore, pheromone trails can be seen as an indirect communication mechanism used by the ants. This system also presents several interesting features from a computational point of view, and has triggered a significant amount of research. The first metaheuristic inspired on this notion was called ant system and was originally proposed for the traveling salesman problem. Over the years, this approach (and its several variations, which are now collectively denominated ant colony optimization or ACO algorithms) has been applied to a wide variety of combinatorial optimization problems, including some pattern recognition tasks (see for example [51]). Nevertheless, its use in multi-objective pattern recognition tasks is very scarce.

\section{Conclusions}

This paper has provided a general overview of multi-objective evolutionary algorithms and some of their possible applications in pattern recognition. In order to make the paper self-contained, a short introduction to evolutionary algorithms has also been provided. After that, the main components that distinguish MOEAs from EAs were discussed, and the main MOEAs in current use were briefly described. In the final part of the paper, some possible paths for future research in this area were discussed. The main aim of this paper is to motivate the development of more research on the use of MOEAs (or any other type of multi-objective metaheuristic) for the solution pattern recognition problems.

\section{Acknowledgements}

The author acknowledges support from CONACyT through project 103570.

\section{References}

1. Miettinen, K.M.: Nonlinear Multiobjective Optimization. Kluwer Academic Publishers, Boston (1999)

2. Blum, C., Roli, A.: Metaheuristics in combinatorial optimization: Overview and conceptual comparison. ACM Computing Surveys 35(3), 268-308 (2003)

3. Coello Coello, C.A., Lamont, G.B., Van Veldhuizen, D.A.: Evolutionary Algorithms for Solving Multi-Objective Problems, 2nd edn. Springer, New York (2007), ISBN 978-0-387-33254-3

4. Goldberg, D.E.: Genetic Algorithms in Search, Optimization and Machine Learning. Addison-Wesley Publishing Company, Reading (1989)

5. Schaffer, J.D.: Multiple Objective Optimization with Vector Evaluated Genetic Algorithms. In: Genetic Algorithms and their Applications: Proceedings of the First International Conference on Genetic Algorithms, pp. 93-100. Lawrence Erlbaum, Mahwah (1985) 
6. Fogel, D.B.: Evolutionary Computation. Toward a New Philosophy of Machine Intelligence. The Institute of Electrical and Electronic Engineers, New York (1995)

7. Holland, J.H.: Concerning efficient adaptive systems. In: Yovits, M.C., Jacobi, G.T., Goldstein, G.D. (eds.) Self-Organizing Systems, pp. 215-230. Spartan Books, Washington, DC (1962)

8. Schwefel, H.P.: Kybernetische evolution als strategie der experimentellen forschung in der strömungstechnik. Dipl.-Ing. thesis (1965) (in German)

9. Fogel, L.J.: Artificial Intelligence through Simulated Evolution. John Wiley, New York (1966)

10. Hajela, P., Lin, C.Y.: Genetic search strategies in multicriterion optimal design. Structural Optimization 4, 99-107 (1992)

11. Srinivas, N., Deb, K.: Multiobjective Optimization Using Nondominated Sorting in Genetic Algorithms. Evolutionary Computation 2(3), 221-248 (1994)

12. Fonseca, C.M., Fleming, P.J.: Genetic Algorithms for Multiobjective Optimization: Formulation, Discussion and Generalization. In: Forrest, S. (ed.) Proceedings of the Fifth International Conference on Genetic Algorithms, University of Illinois at Urbana-Champaign, San Mateo,California, pp. 416-423. Morgan Kauffman Publishers, San Francisco (1993)

13. Beume, N., Naujoks, B., Emmerich, M.: SMS-EMOA: Multiobjective selection based on dominated hypervolume. European Journal of Operational Research 181(3), 1653-1669 (2007)

14. Goldberg, D.E., Richardson, J.: Genetic algorithm with sharing for multimodal function optimization. In: Grefenstette, J.J. (ed.) Genetic Algorithms and Their Applications: Proceedings of the Second International Conference on Genetic Algorithms, pp. 41-49. Lawrence Erlbaum, Hillsdale (1987)

15. Deb, K., Goldberg, D.E.: An Investigation of Niche and Species Formation in Genetic Function Optimization. In: Schaffer, J.D. (ed.) Proceedings of the Third International Conference on Genetic Algorithms, George Mason University, San Mateo, California, June 1989, pp. 42-50. Morgan Kaufmann, San Francisco (1989)

16. Toscano Pulido, G., Coello Coello, C.A.: Using clustering techniques to improve the performance of a multi-objective particle swarm optimizer. In: Deb, K., et al. (eds.) GECCO 2004. LNCS, vol. 3102, pp. 225-237. Springer, Heidelberg (2004)

17. Zitzler, E., Deb, K., Thiele, L.: Comparison of Multiobjective Evolutionary Algorithms on Test Functions of Different Difficulty. In: Wu, A.S. (ed.) Proceedings of the 1999 Genetic and Evolutionary Computation Conference, Workshop Program, Orlando, Florida, July 1999, pp. 121-122 (1999)

18. Knowles, J., Corne, D.: Properties of an Adaptive Archiving Algorithm for Storing Nondominated Vectors. IEEE Transactions on Evolutionary Computation 7(2), 100-116 (2003)

19. Kita, H., Yabumoto, Y., Mori, N., Nishikawa, Y.: Multi-Objective Optimization by Means of the Thermodynamical Genetic Algorithm. In: Ebeling, W., Rechenberg, I., Voigt, H.-M., Schwefel, H.-P. (eds.) PPSN 1996. LNCS, vol. 1141, pp. 504-512. Springer, Heidelberg (1996)

20. Cui, X., Li, M., Fang, T.: Study of Population Diversity of Multiobjective Evolutionary Algorithm Based on Immune and Entropy Principles. In: Proceedings of the Congress on Evolutionary Computation 2001 (CEC 2001), May 2001, vol. 2, pp. 1316-1321. IEEE Service Center, Piscataway (2001)

21. Knowles, J.D., Corne, D.W.: Approximating the Nondominated Front Using the Pareto Archived Evolution Strategy. Evolutionary Computation 8(2), 149-172 (2000) 
22. Zitzler, E., Laumanns, M., Thiele, L.: SPEA2: Improving the Strength Pareto Evolutionary Algorithm. In: Giannakoglou, K., Tsahalis, D., Periaux, J., Papailou, P., Fogarty, T. (eds.) EUROGEN 2001 Evolutionary Methods for Design, Optimization and Control with Applications to Industrial Problems, Athens, Greece, pp. 95-100 (2001)

23. Zhang, Q., Li, H.: MOEA/D: A Multiobjective Evolutionary Algorithm Based on Decomposition. IEEE Transactions on Evolutionary Computation 11(6), 712-731 (2007)

24. Deb, K., Mohan, M., Mishra, S.: Evaluating the $\epsilon$-Domination Based MultiObjective Evolutionary Algorithm for a Quick Computation of Pareto-Optimal Solutions. Evolutionary Computation 13(4), 501-525 (2005)

25. Toscano Pulido, G., Coello Coello, C.A.: The micro genetic algorithm 2: Towards online adaptation in evolutionary multiobjective optimization. In: Fonseca, C.M., Fleming, P.J., Zitzler, E., Deb, K., Thiele, L. (eds.) EMO 2003. LNCS, vol. 2632, pp. 252-266. Springer, Heidelberg (2003)

26. Knowles, J.: ParEGO: A Hybrid Algorithm With On-Line Landscape Approximation for Expensive Multiobjective Optimization Problems. IEEE Transactions on Evolutionary Computation 10(1), 50-66 (2006)

27. Chin-Wei, B., Rajeswari, M.: Multiobjective Optimization Approaches in Image Segmentation-The Directions and Challenges. In: International on Advances in Soft Computing and its Applications, March 2010, vol. 2(1), pp. 40-65 (2010)

28. Bhanu, B., Lee, S.: Genetic Learning for Adaptive Image Segmentation. Kluwer Academic Publishers, Boston (1994)

29. Shirakawa, S., Nagao, T.: Evolutionary Image Segmentation Based on Multiobjective Clustering. In: 2009 IEEE Congress on Evolutionary Computation (CEC 2009), Trondheim, Norway, May 2009, pp. 2466-2473. IEEE Press, Los Alamitos (2009)

30. Hamdani, T.M., Won, J.-M., Alimi, M.A.M., Karray, F.: Multi-objective feature selection with NSGA II. In: Beliczynski, B., Dzielinski, A., Iwanowski, M., Ribeiro, B. (eds.) ICANNGA 2007. LNCS, vol. 4431, pp. 240-247. Springer, Heidelberg (2007)

31. Dasarathy, B.V.: Nearest Neighbor (NN) Norms: NN Pattern Classification Techniques. IEEE Computer Society Press, Los Alamitos (1990)

32. Deb, K., Pratap, A., Agarwal, S., Meyarivan, T.: A Fast and Elitist Multiobjective Genetic Algorithm: NSGA-II. IEEE Transactions on Evolutionary Computation 6(2), 182-197 (2002)

33. Morita, M., Sabourin, R., Bortolozzi, F., Suen, C.: Unsupervised Feature Selection Using Multi-Objective Genetic Algorithm for Handwritten Word Recognition. In: Proceedings of the 7th International Conference on Document Analysis and Recognition (ICDAR 2003), Edinburgh, Scotland, August 2003, pp. 666-670 (2003)

34. Emmanouilidis, C., Hunter, A., MacIntyre, J.: A Multiobjective Evolutionary Setting for Feature Selection and a Commonality-Based Crossover Operator. In: 2000 Congress on Evolutionary Computation, July 2000, vol. 1, pp. 309-316. IEEE Computer Society Press, Piscataway (2000)

35. Zaliz, R.R., Zwir, I., Ruspini, E.: Generalized Analysis of Promoters: A Method for DNA Sequence Description. In: Coello Coello, C.A., Lamont, G.B. (eds.) Applications of Multi-Objective Evolutionary Algorithms, pp. 427-449. World Scientific, Singapore (2004) 
36. de la Iglesia, B., Reynolds, A., Rayward-Smith, V.J.: Developments on a multiobjective metaheuristic (MOMH) algorithm for finding interesting sets of classification rules. In: Coello Coello, C.A., Hernández Aguirre, A., Zitzler, E. (eds.) EMO 2005. LNCS, vol. 3410, pp. 826-840. Springer, Heidelberg (2005)

37. de la Iglesia, B., Richards, G., Philpott, M., Rayward-Smith, V.: The application and effectiveness of a multi-objective metaheuristic algorithm for partial classification. European Journal of Operational Research 169, 898-917 (2006)

38. Bandyopadhyay, S., Pal, S.K., Aruna, B.: Multiobjective GAs, Quantitative Indices, and Pattern Classification. IEEE Transactions on Systems, Man, and Cybernetics-Part B: Cybernetics 34(5), 2088-2099 (2004)

39. Guo, P.F., Bhattacharya, P., Kharma, N.: An Efficient Image Pattern Recognition System Using an Evolutionary Search Strategy. In: Proceedings of the 2009 IEEE International Conference on Systems, Man, and Cybernetics, October 2009. IEEE Press, San Antonio (2009)

40. Koza, J.R.: Genetic Programming. On the Programming of Computers by Means of Natural Selection. The MIT Press, Cambridge (1992)

41. Jin, Y.: A comprehensive survey of fitness approximation in evolutionary computation. Soft Computing 9(1), 3-12 (2005)

42. López Jaimes, A., Coello Coello, C.A.: Applications of Parallel Platforms and Models in Evolutionary Multi-Objective Optimization. In: Lewis, A., Mostaghim, S., Randall, M. (eds.) Biologically-Inspired Optimisation Methods, pp. 23-49. Springer, Heidelberg (2009), ISBN 978-3-642-01261-7

43. Lim, D., Jin, Y., Ong, Y.S., Sendhoff, B.: Generalizing Surrogate-Assisted Evolutionary Computation. IEEE Transactions on Evolutionary Computation 14(3), 329-355 (2010)

44. Corne, D., Dorigo, M., Glover, F. (eds.): New Ideas in Optimization. McGraw-Hill, London (1999)

45. Kennedy, J., Eberhart, R.C.: Swarm Intelligence. Morgan Kaufmann Publishers, California (2001)

46. Chander, A., Chatterjee, A., Siarry, P.: A new social and momentum component adaptive PSO algorithm for image segmentation. Expert Systems with Applications 38(5), 4998-5004 (2011)

47. Dasgupta, D. (ed.): Artificial Immune Systems and Their Applications. Springer, Berlin (1999)

48. Nunes de Castro, L., Timmis, J.: An Introduction to Artificial Immune Systems: A New Computational Intelligence Paradigm. Springer, London (2002)

49. Wang, W., Gao, S., Tang, Z.: Improved pattern recognition with complex artificial immune system. Soft Computing 13(12), 1209-1217 (2009)

50. Dorigo, M., Stützle, T.: Ant Colony Optimization. The MIT Press, Cambridge (2004), ISBN 0-262-04219-3

51. Tambouratzis, G.: Using an Ant Colony Metaheuristic to Optimize Automatic Word Segmentation for Ancient Greek. IEEE Transactions on Evolutionary Computation 13(4), 742-753 (2009) 